\title{
WITH RESPECT TO TAIL SIGMA FIELDS, STANDARD MEASURES POSSESS MEASURABLE DISINTEGRATIONS
}

\author{
LESTER E. DUBINS ${ }^{1}$ AND DAVID HEATH
}

\begin{abstract}
Let $P$ be a countably additive probability on a standard space, and let $\mathbb{A}$ be a tail subfield. Though no disintegration of $P$ with respect to $Q$ that is countably additive need exist, there always is one which is finitely additive.
\end{abstract}

A surprising discovery of de Finetti, [5, p. 205] (see also [4]), leads to the conclusion that probability measures which are not countably additive need not possess any disintegration with respect to certain partitions. Even countably additive, fair-coin-tossing measure possesses no proper, measurable, countably additive disintegration with respect to the usual tail field [3]. One of the questions that then arises concerns the existence of any disintegration for this and similar situations. For standard measures and tail fields, an affirmative answer is provided by the theorem below. But first a little notation and two or three definitions are needed.

Throughout this note, $\mathbb{Q}$ is a subsigma field of a sigma field $\mathscr{B}$ of subsets of a set $\Omega$, and $P$ is a countably additive probability defined for $\Re$. For each $\omega \in \Omega$, let $Q(\omega)$ be the intersection of all $A$ such that $\omega \in A \in \mathbb{Q}$. Whether or not $\mathbb{Q}(\omega) \in \mathbb{Q}$, call $\mathbb{Q}(\omega)$ an $\mathcal{Q}$-atom, and verify that the $Q$-atoms constitute a partition $\Pi_{\mathscr{Q}}$ of $\Omega$. A function $\sigma$ of two variables, $h \in \Pi_{\mathfrak{Q}}, B \in \Re$ is a measurable disintegration of $P$ given $Q$ if:

(i) For each $h, \sigma(\cdot \mid h)$ is a probability on $\mathscr{B}$, possibly finitely additive, which is supported by $h$;

(ii) For each $B, \sigma(B \mid \mathcal{Q}(\omega))$ is a version of the usual $P$-conditional-expectation of $B$ given $Q$.

A tail field is the intersection of a decreasing sequence of subsigma fields of $\mathscr{B}$ each of which is countably generated.

THEOREM 1. Let $(\Omega, \Re)$ be a standard space or, more generally, a Lusin space, and let $\mathbb{Q}$ be a tail subfield of $\Re$. Then every countably additive probability, $P$, on $\Re$ possesses a measurable disintegration given $Q$.

If the conclusion of Theorem 1 holds for $\mathbb{Q}$, call $\mathbb{Q}$ tame.

LEMmA 1. Countably generated $\mathbb{Q}$ 's in Lusin spaces are tame.

Received by the editors July 6, 1982 and, in revised form, October 6, 1982.

1980 Mathematics Subject Classification. Primary 28A35, 28A50, 60G05.

Key words and phrases. Conglomerability, disintegrations, finite additivity.

${ }^{1}$ Research supported by NSF Grant MCS-80-02535. 
Proof. In a stronger form, this assertion is contained in [2, Theorem 2] which, in turn, was seen as a simple corollary to [1, Theorem 5].

In view of Lemma 1 , to establish Theorem 1, it suffices to establish

PROPOSITION 1. The intersection of a decreasing sequence of sigma fields, each of which is tame, is tame.

It is helpful to restate Proposition 1 in a more informative form, as in the next lemma, in which a diffuse mixture of disintegrations plays a role. A probability on the integers is diffuse if each finite set is of probability zero.

LEMMA 2. Let $P$ be a countably additive probability on $\mathscr{B}, Q$ the intersection of $a$ decreasing sequence of tame fields $\mathbb{Q}_{n}, \sigma_{n}$ a measurable disintegration of $P$ given $\mathcal{Q}_{n}, \mu$ a diffuse probability on the positive integers, and $\sigma_{\mu}$ defined for $B \in \mathscr{G}, \omega \in \Omega$ by

$$
\sigma_{\mu}(B \mid \mathcal{Q}(\omega))=\int \sigma_{n}\left(B \mid \mathbb{Q}_{n}(\omega)\right) \mu(d n) .
$$

Then $\sigma_{\mu}$ is a measurable disintegration of $P$ given $Q$.

The proof of Lemma 2 requires two preliminary facts.

Schоlium 1. Let $Q$ be the intersection of sigma fields $\Theta_{1} \supset Q_{2} \supset \cdots$, and let $A_{n} \in Q_{n}$. Then $\liminf A_{n} \in Q$.

Proof. Let $B_{j}=\bigcap A_{n}(n \geqslant j)$. Plainly, since $B_{i} \in \mathbb{Q}_{i} \subset \mathbb{Q}_{j}$ for $i>j, \cup B_{i}(i \geqslant j)$ $\in \mathbb{Q}_{j}$. And since this union is $\liminf A_{n}$, independent of $j$, it belongs to every $\mathbb{Q}_{j}$, that is, it belongs to $Q$.

SchоliUm 2. If $Q$ is the intersection of sigma fields $\Theta_{1} \supset Q_{2} \supset \cdots$, then, for each $\omega \in \Omega$,

$$
Q(\omega)=\cup Q_{n}(\omega) \text {. }
$$

Proof. Since $Q \subset Q_{n}, Q(\omega) \supset Q_{n}(\omega)$ for all $n$, the left-hand side of (2) includes the right-hand side. It suffices to see, therefore, that unless $\omega^{\prime} \in \Omega$ belongs to one of the $\mathbb{Q}_{n}(\omega)$, it does not belong to $\mathbb{Q}(\omega)$. So assume that $\exists A_{n} \in \mathbb{Q}_{n}$ which separates $\omega$ from $\omega^{\prime}$, say $\omega \in A_{n}, \omega^{\prime} \in A_{n}^{c}$ for all $n$. Let $A=\liminf A_{n}$. Then $A$ obviously separates $\omega$ from $\omega^{\prime}$ and, by Scholium $1, A \in \mathbb{Q}$. So $\omega^{\prime} \notin \mathbb{Q}(\omega)$.

Proof of Lemma 2. If $\mathbb{Q}(\omega)=\mathbb{Q}\left(\omega^{\prime}\right)$, then, by Scholium 2, $\mathbb{Q}_{n}(\omega)=\mathbb{Q}_{n}\left(\omega^{\prime}\right)$ for some $n$ and, hence, for all but a finite set of $n$. So, since $\mu$ is diffuse, $\sigma_{\mu}(B \mid \omega)=$ $\sigma_{\mu}\left(B \mid \omega^{\prime}\right)$, that is, $\sigma_{\mu}(\cdot \mid \omega)$ depends only on $\mathcal{Q}(\omega)$, and may therefore be written as $\sigma_{\mu}(\cdot \mid \mathbb{Q}(\omega))$. Since $\sigma_{n}\left(\cdot \mid \mathbb{Q}_{n}(\omega)\right)$ is supported by $\mathbb{Q}_{n}(\omega)$, and $\mathbb{Q}_{n}(\omega) \subset \mathbb{Q}(\omega)$, it is, for all $n$, certainly supported by $\mathcal{Q}(\omega)$.

Therefore, $\sigma_{\mu}(\cdot \mid \mathcal{Q}(\omega))$, too, is supported by $\mathscr{Q}(\omega)$. Hence, (i) is satisfied by $\sigma_{\mu}$. To verify (ii), note that $\sigma_{n}\left(B \mid Q_{n}(\omega)\right)$, being a version of the $P$-conditional probability of $B$ given $\mathcal{Q}_{n}$, converges, by the reversed martingale convergence theorem, for all $\omega$ not in a $P$-null set, to a limit $X(\omega)$, where $X$ is a version of $P(B \mid \mathcal{Q})$. Since $\mu$ is a diffuse probability, for all such $\omega$, the right-hand side of (1) is $X(\omega)$. So $\sigma_{\mu}(B \mid Q(\omega))$ satisfies (ii). 
Plainly, Theorem 1 implies that countably additive probabilities possess measurable disintegrations given the usual tail fields as well as fields of the form $\mathscr{F}_{t+}$ familiar in the usual theory of continuous-path processes. The same conclusion holds for the subfield of Borel sets invariant under a group which is the union of an increasing sequence of finite groups as is, for example, the group of periodic rotations of a circle. In particular, the usual fair-coin probability possesses a measurable disintegration given the tail sigma-field which, at the time [3] was written, seemed unlikely. Since, as reported there, all such disintegrations are purely finitely additive, the conclusion of Theorem 1 cannot be strengthened to assert the existence of measurable disintegrations which are countably additive. That the hypothesis that $P$ is countably additive cannot be dropped can be seen by means of an example [4, Theorem 2].

Whether the conclusion of Theorem 1 holds if $\mathbb{Q}$ consists of the Borel subsets of the unit circle invariant under a nonperiodic rotation, we do not know. Indeed, whether the hypothesis of Theorem 1, that $Q$ be a tail field, can be considerably weakened, if not entirely eliminated, we must leave open. To us, this seems dubious even if "measurable" is deleted from the conclusion.

\section{REFERENCES}

1. David Blackwell, On a class of probability spaces, Proc. Third Berkeley Sympos. Math. Statist. and Prob. 1-6, University of California Press, Berkeley, Calif., 1955.

2. David Blackwell and Lester E. Dubins, On existence and non-existence of proper, regular, conditional distributions, Ann. Probab. 3 (1975), 741-752.

3. Lester E. Dubins, Measurable, tail disintegrations of the Haar integral are purely finitely additive, Proc. Amer. Math. Soc. 62 (1977), 34-36.

4. Finitely additive conditional probabilities, conglomerability and disintegrations, Ann. Probab. 3 (1975), 89-99.

5. B. de Finetti, Probability, induction, and statistics, Wiley, New York, 1972.

Department of Mathematics, University of California, Berkeley, California 94720

DEPARTMENT OF OPERATIONS RESEARCH AND INDUSTRIAL ENGINEERING, CORNELL UNIVERSITY, ITHACA, NEW YORK 14853 